\title{
$\begin{array}{ll}\text { Research Square } & \begin{array}{l}\text { Preprints are preliminary reports that have not undergone peer review. } \\ \text { They should not be considered conclusive, used to inform clinical practice, } \\ \text { or referenced by the media as validated information. }\end{array}\end{array}$
}

\section{The incidence of stroke among patients undergoing elective posterior lumbar fusion: a retrospective cohort study}

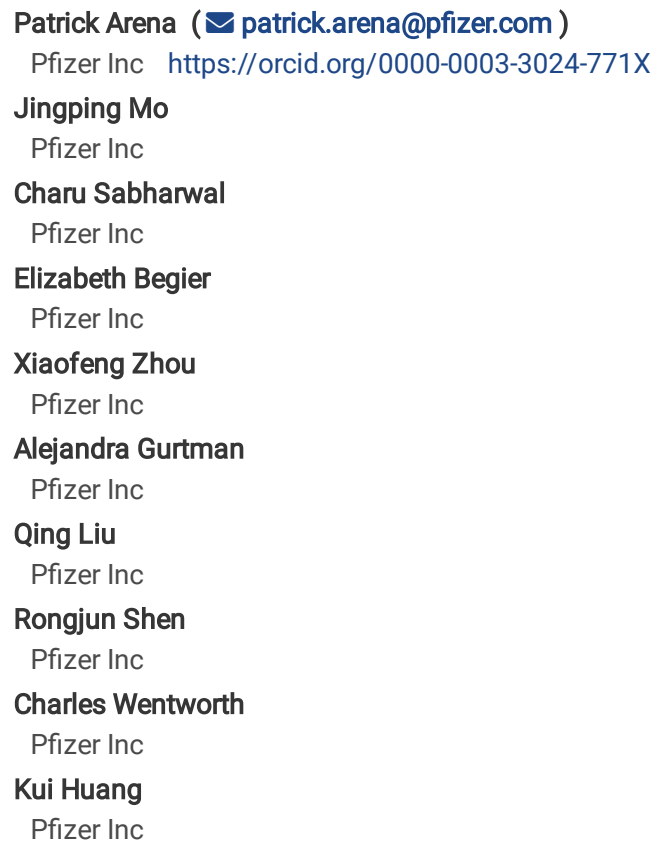

Version of Record: A version of this preprint was published on September 14th, 2020. See the published version at https://doi.org/10.1186/s12891-020-036315. 


\section{Abstract}

Background: Although stroke is an uncommon but life-threatening complication among spinal surgery patients, the recognition of this adverse event is critical given the aging population undergoing surgical procedures. The objective of this study was to estimate the incidence of stroke among adults undergoing elective posterior lumbar fusion (PLF) during post-operative risk windows and among different subgroups.

Methods: A retrospective cohort study using a longitudinal electronic healthcare record (EHR) database was conducted from January 1, 2007 to June 30 , 2018. Elective PLF, stroke, and select clinical characteristics were defined based on International Classification of Disease codes. Patients aged 18 to 85 years with $\geq 183$ days of enrollment in the database prior to undergoing elective PLF were followed from the index date until the occurrence of stroke, death, loss to follow-up, or end of study period, whichever occurred first. Incidence proportions (IPs) and rates (IRs) of stroke were estimated in the following risk windows: index hospitalization, $\leq 30$ days, $\leq 90$ days, $\leq 180$ days, and $\leq 365$ days post-operation

Results: A total of 43,063 patients were eligible for the study. The IP of stroke following elective PLF ranged from $0.29 \%$ ( $95 \%$ confidence interval [CI]: $0.25 \%$, $0.35 \%)$ during index hospitalization to $1.12 \%(95 \% \mathrm{Cl}: 1.03 \%, 1.23 \%) \leq 365$ days post-operation; the IR of stroke following elective PLF per 1000 person-years decreased consistently from 229.08 (95\% Cl: $192.38,272.78)$ during index hospitalization to 13.68 (95\% Cl: $12.51,14.96) \leq 365$ days post-operation. Stratified analyses revealed that older patients had a higher incidence of stroke. Additionally, black patients had higher stroke incidences than white, Asian, or other patients. Furthermore, the incidence of post-operative stroke was higher among patients with a history of type 2 diabetes than among patients without such history.

Conclusions: The incidence of stroke following elective PLF found here using an EHR database is slightly higher than that reported in the literature; however, the discrepancy is due to differences in the variable definitions, study populations, follow-up periods, and data sources between our study and those in the literature.

\section{Introduction}

Stroke was the second leading cause of death globally in 2016, with 177,196 stroke deaths and 731,256 incident cases in the United States (US) alone [1]. Although stroke is an uncommon but life-threatening complication among spinal surgery patients [2], the recognition of this adverse event is critical given the aging population undergoing surgical procedures. The volume of elective lumbar fusion procedures in the US has increased $62.3 \%$, from 122,679 cases in 2004 to 199,140 cases in 2015; these increases were greatest among those aged 65 years or older [3]. Furthermore, Etzioni et al. have demonstrated that the number of older people (i.e., those above the age of 65 years) undergoing surgery in general is increasing at a rate faster than the proportion of older people in the overall population [4]. For adults undergoing posterior lumbar fusions (PLFs), the incidence of stroke within 30 days post-surgery has been reported as approximately $0.2 \%$ [5-8], but there is little data during various post-operative risk windows.

It is important to have background epidemiology data about post-operative stroke during various risk windows and among different subgroups to contextualize safety data in clinical trials and the general population. This cohort study was thus designed to estimate the incidence of stroke among adults undergoing elective PLF using a large electronic healthcare record (EHR) database in the US. The objectives of this study were to estimate the incidence proportions (IPs) and incidence rates (IRs) of stroke among elective PLF patients in various post-operative time periods (as well as during the index surgical hospitalization) and within different subgroups.

\section{Methods}

\section{Study design}

A retrospective cohort study of adults undergoing elective PLF using Optum EHR, a longitudinal US EHR database, was performed. The study period was January 1, 2007 to June 30, 2018 with a length of follow-up equating to 365 days. The index surgical date was defined as the first date on or after January 1 , 2007 that an adult had undergone an elective PLF. To incorporate a 183-day look-back window prior to the index surgery (for the purpose of excluding prevalent conditions), the earliest possible index surgical date was July 1, 2007. The latest index surgical date possible was June $30,2017$.

\section{Cohort formation}

Eligible patients were 18 to 85 years of age at the time of their first elective PLF. Furthermore, patients had 183 days of continuous enrollment within the database prior to their first elective PLF (i.e., the baseline period) as well as this index surgery being performed on the day of admission to the healthcare facility or the day after admission. PLF was identified using the following six International Classification of Diseases (ICD), Ninth Revision, Procedure Classification System (ICD-9-PCS) codes: 81.05, 81.07, 81.08, 81.35, 81.37, and 81.38; these codes referred to lumbar fusion and refusion of either the posterior or anterior column using the posterior technique.

To ensure a healthy study cohort and select for elective surgeries, patients were excluded if they 1) underwent a major surgical procedure that occurred within 90 days prior to the index surgery; 2) had a surgical indication that was for an emergency procedure; 3 ) were pregnant; 4) were discharged on the same date of the index surgery (thereby indicating an outpatient procedure); 5) had any of the following conditions during the baseline period: anaphylactic reaction to a vaccine, cancer, end stage renal disease, congenital spleen anomalies, an immunosuppressive state, and/or receipt of corticosteroids or immunosuppressive medications; or 6) had any of the following conditions time of the index surgery: potential/presumed surgical site-related infection and/or spinal infection.

Stroke was defined based on the following ten ICD, Ninth Revision, Clinical Modification (ICD-9-CM) codes: 433.01, 433.11, 433.21, 433.31, 433.81, 433.91, $434.01,434.11,434.91$, and 436 ; these codes referred to occlusion, stenosis, thrombosis, and embolism of arteries with cerebral infraction as well as cerebral 
artery occlusion and acute cerebrovascular disease. ICD-9-PCS and ICD-9-CM codes were mapped to corresponding ICD, Tenth Revision (ICD-10) codes using General Equivalence Mapping techniques in order to account for the switch to ICD-10 coding in 2015.

\section{Data management and analysis}

All analyses were descriptive and conducted in SAS (version 9.4, SAS Institute, Cary, NC, USA). Descriptive statistics were performed to characterize the cohort in terms of demographic and clinical characteristics at the baseline. Patients were followed from the cohort entry index date until the occurrence of stroke, death, loss to follow-up, or end of study period, whichever occurred first.

IPs and IRs were estimated with associated $95 \%$ confidence intervals (Cls), assuming a Poisson distribution. Incidences were calculated overall, and in the following subgroups: age, gender, race, length of hospital stay, and selected clinical characteristics. Incidences were estimated in the following risk windows: index hospitalization (defined as the time interval from index surgery to discharge), $\leq 30$ days (i.e., 0 to 30 days), $\leq 90$ days (i.e., 0 to 90 days), $\leq 180$ days (i.e., 0 to 180 days), and $\leq 365$ days (i.e., 0 to 365 days) post-operation.

\section{Results}

Of the 80,796 patients who were 18 to 85 years of age with at least one record of elective PLF during the study period and adequate prior enrollment in the database, 37,733 met exclusion criteria; the most common reasons for exclusion were use of immunosuppressive medications (42\%), receipt of systemic corticosteroids (33\%), and a diagnosis of cancer (14\%) during the baseline. Ultimately, 43,063 patients were included. The mean age was 59.4 years, and there were slightly more females (52.22\%) than males (47.75\%). The majority of the cohort members were white (89.49\%), while black (5.94\%) and Asian (0.52\%) members were less represented. The most prevalent medical conditions were type 2 diabetes (13.86\%), cardiac dysrhythmias (9.28\%), and chronic ischemic disease $(9.00 \%)$. Only 293 patients $(0.68 \%)$ had any history of stroke during the baseline. Dementia, individual digestive disorders, deep vein thrombosis, and pulmonary embolism were also rare (i.e., each less than 1.00\%). Lastly, the average length of hospital stay (for the index hospitalization) was 3.8 days. Table 1 shows the baseline demographics and clinical characteristics of the elective PLF patient population. 
Table 1

Baseline demographics, clinical characteristics, and surgical characteristics of the elective posterior lumbar fusion patient population

\begin{tabular}{|c|c|c|}
\hline Characteristic, $N(\%)$ except where specified & Number of patients & $\%$ \\
\hline Total & 43,063 & \\
\hline \multicolumn{3}{|l|}{ Demographic characteristics } \\
\hline \multicolumn{3}{|l|}{ Age at index date } \\
\hline Mean (SD) & $59.4(13.69)$ & \\
\hline Median (Range) & $61(18,85)$ & \\
\hline$>=18-55$ & 15,221 & 35.35 \\
\hline $56-65$ & 11,639 & 27.03 \\
\hline $66-75$ & 11,579 & 26.89 \\
\hline $76-<86$ & 4,624 & 10.74 \\
\hline \multicolumn{3}{|l|}{ Race } \\
\hline White & 38,535 & 89.49 \\
\hline Black or African American & 2,556 & 5.94 \\
\hline Asian & 223 & 0.52 \\
\hline Other/Unknown & 1,749 & 4.06 \\
\hline \multicolumn{3}{|l|}{ Sex } \\
\hline Male & 20,563 & 47.75 \\
\hline Female & 22,487 & 52.22 \\
\hline Unknown & 13 & 0.03 \\
\hline \multicolumn{3}{|l|}{ Clinical \& surgical characteristics in 183 -day baseline } \\
\hline \multicolumn{3}{|l|}{ Arthritis and other inflammation (or rheumatic events) } \\
\hline Rheumatoid arthritis & 0 & 0.00 \\
\hline Reactive arthritis & 579 & 1.34 \\
\hline Psoriatic arthroplasty & 42 & 0.10 \\
\hline Spondyloarthritis, including ankylosing spondylitis & 1,476 & 3.43 \\
\hline \multicolumn{3}{|l|}{ Blood disorders } \\
\hline Anemia/other anemia & 3,494 & 8.11 \\
\hline Intracranial Hemorrhage & 52 & 0.12 \\
\hline Gastrointestinal bleeding & 216 & 0.50 \\
\hline Thrombocytopenia & 333 & 0.77 \\
\hline \multicolumn{3}{|l|}{ Cardiovascular events and/or conditions } \\
\hline Acute myocardial infarction & 270 & 0.63 \\
\hline Angina & 1,997 & 4.64 \\
\hline Cardiac dysrhythmias & 3,997 & 9.28 \\
\hline Stroke & 293 & 0.68 \\
\hline Chronic ischemic disease & 3,876 & 9.00 \\
\hline Peripheral vascular disease & 1,811 & 4.21 \\
\hline \multicolumn{3}{|l|}{ Dementia } \\
\hline Dementia & 130 & 0.30 \\
\hline \multicolumn{3}{|l|}{ Diabetes } \\
\hline Diabetes, type 1 & 272 & 0.63 \\
\hline
\end{tabular}




\begin{tabular}{|c|c|c|}
\hline Characteristic, N (\%) except where specified & Number of patients & $\%$ \\
\hline Diabetes, type 2 & 5,970 & 13.86 \\
\hline \multicolumn{3}{|l|}{ Digestive disorders } \\
\hline Crohn's disease & 73 & 0.17 \\
\hline Ulcerative colitis & 71 & 0.16 \\
\hline Peptic ulcer disease & 240 & 0.56 \\
\hline \multicolumn{3}{|l|}{ Hepatic disorders } \\
\hline Liver disease and cirrhosis & 607 & 1.41 \\
\hline \multicolumn{3}{|l|}{ Nervous system disorders } \\
\hline Guillain-Barre syndrome & 0 & 0.00 \\
\hline Multiple sclerosis & 105 & 0.24 \\
\hline \multicolumn{3}{|l|}{ Respiratory disorders } \\
\hline Asthma/wheezing/bronchospasm & 3,353 & 7.79 \\
\hline Bronchitis/chronic obstructive pulmonary disease & 2,315 & 5.38 \\
\hline Obstructive asthma & 1,126 & 2.61 \\
\hline \multicolumn{3}{|l|}{ Surgical characteristics } \\
\hline Existing permanently implanted device or prosthesis at baseline & 1,270 & 2.95 \\
\hline \multicolumn{3}{|l|}{ Total length of hospital stay } \\
\hline Mean (SD) & $3.8(3.4)$ & \\
\hline Median (Min, Max) & $3(1,90)$ & \\
\hline History of allogenic blood transfusion during surgery & 1 & 0.00 \\
\hline Revisional surgery on the same day as index surgery & 320 & 0.74 \\
\hline Use of implanted material during surgery on the same day as index surgery & 2,667 & 6.19 \\
\hline \multicolumn{3}{|l|}{ Thrombolic events } \\
\hline Deep vein thrombosis & 386 & 0.90 \\
\hline Pulmonary embolism & 170 & 0.39 \\
\hline \multicolumn{3}{|l|}{ Thyroid disorders } \\
\hline Grave's disease & 37 & 0.09 \\
\hline Autoimmune thyroiditis & 55 & 0.13 \\
\hline Hyperthyroidism & 109 & 0.25 \\
\hline Hypothyroidism & 3,455 & 8.02 \\
\hline Thyroiditis & 6 & 0.01 \\
\hline
\end{tabular}

[Insert Table 1 here]

The IP of stroke following elective PLF was $0.29 \%$ (95\% Cl: $0.25 \%, 0.35 \%)$ during index hospitalization, $0.44 \%(95 \% \mathrm{Cl}$ : $0.38 \%, 0.50 \%) \leq 30$ days, $0.59 \%$ (95\% Cl: $0.52 \%, 0.67 \%) \leq 90$ days, $0.76 \%$ (95\% Cl: $0.68 \%, 0.85 \%) \leq 180$ days, and $1.12 \%(95 \% \mathrm{Cl}: 1.03 \%, 1.23 \%) \leq 365$ days post-operation. The IR of stroke following elective PLF decreased consistently from index hospitalization to $\leq 365$ days post-operation; these IRs per 1000 person-years (PYs) were 229.08

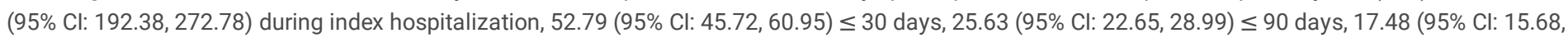
$19.48) \leq 180$ days, and $13.68(95 \% \mathrm{Cl}: 12.51,14.96) \leq 365$ days post-operation. Tables 2 and 3 show the IP and IR of stroke in patients undergoing elective PLF, respectively. 
Table 2

Incidence proportion of stroke in patients undergoing elective posterior lumbar fusi

\begin{tabular}{|c|c|c|c|c|c|c|c|c|c|c|c|c|}
\hline \multirow{2}{*}{$\begin{array}{l}\text { Stroke } \\
\text { incidence type }\end{array}$} & \multicolumn{4}{|c|}{ During index hospitalization } & \multicolumn{4}{|c|}{ Up to 30 days post-operation } & \multicolumn{4}{|c|}{ Up to 90 days post-operation } \\
\hline & $\begin{array}{l}\text { Number } \\
\text { of } \\
\text { patients } \\
\text { at risk }\end{array}$ & $\begin{array}{l}\text { Number } \\
\text { of } \\
\text { cases }\end{array}$ & $\begin{array}{l}\text { Years } \\
\text { at risk }\end{array}$ & $\begin{array}{l}\text { Incidence } \\
\text { proportion } \\
\text { ( } 95 \% \\
\text { confidence } \\
\text { interval) }\end{array}$ & $\begin{array}{l}\text { Number } \\
\text { of } \\
\text { patients } \\
\text { at risk }\end{array}$ & $\begin{array}{l}\text { Number } \\
\text { of } \\
\text { cases }\end{array}$ & $\begin{array}{l}\text { Years at } \\
\text { risk }\end{array}$ & $\begin{array}{l}\text { Incidence } \\
\text { proportion } \\
\text { (95\% } \\
\text { confidence } \\
\text { interval) }\end{array}$ & $\begin{array}{l}\text { Number } \\
\text { of } \\
\text { patients } \\
\text { at risk }\end{array}$ & $\begin{array}{l}\text { Number } \\
\text { of } \\
\text { cases }\end{array}$ & $\begin{array}{l}\text { Years at } \\
\text { risk }\end{array}$ & $\begin{array}{l}\text { Incide } \\
\text { propol } \\
\text { (95\% } \\
\text { confid } \\
\text { interv: }\end{array}$ \\
\hline Crude & 42,742 & 126 & 550.03 & $\begin{array}{l}0.29 \% \\
(0.25 \% \\
0.35 \%)\end{array}$ & 42,744 & 186 & 3523.41 & $\begin{array}{l}0.44 \% \\
(0.38 \%, \\
0.50 \%)\end{array}$ & 42,745 & 252 & 9833.32 & $\begin{array}{l}0.59 \% \\
(0.52 \% \\
0.67 \%\end{array}$ \\
\hline \multicolumn{13}{|l|}{$\begin{array}{l}\text { Strafications } \\
\text { by: }\end{array}$} \\
\hline \multicolumn{13}{|l|}{ Age } \\
\hline$>=18-55$ & 15,181 & 17 & 179.62 & $\begin{array}{l}0.11 \% \\
(0.07 \% \\
0.18 \%)\end{array}$ & 15,181 & 25 & 1251.95 & $\begin{array}{l}0.16 \% \\
(0.11 \% \\
0.24 \%)\end{array}$ & 15,181 & 36 & 3498.67 & $\begin{array}{l}0.24 \% \\
(0.17 \% \\
0.33 \%\end{array}$ \\
\hline $56-65$ & 11,588 & 22 & 146.04 & $\begin{array}{l}0.19 \% \\
(0.12 \% \\
0.29 \%)\end{array}$ & 11,590 & 39 & 956.04 & $\begin{array}{l}0.34 \% \\
(0.24 \% \\
0.46 \%)\end{array}$ & 11,591 & 53 & 2662.86 & $\begin{array}{l}0.46 \% \\
(0.34 \% \\
0.60 \%\end{array}$ \\
\hline $66-75$ & 11,438 & 51 & 157.62 & $\begin{array}{l}0.45 \% \\
(0.33 \% \\
0.59 \%)\end{array}$ & 11,438 & 71 & 942.84 & $\begin{array}{l}0.62 \% \\
(0.49 \% \\
0.78 \%)\end{array}$ & 11,438 & 90 & 2634.55 & $\begin{array}{l}0.79 \% \\
(0.63 \% \\
0.97 \%\end{array}$ \\
\hline $76-<86$ & 4,535 & 36 & 66.74 & $\begin{array}{l}0.79 \% \\
(0.56 \%, \\
1.10 \%)\end{array}$ & 4,535 & 51 & 372.57 & $\begin{array}{l}1.12 \% \\
(0.84 \%, \\
1.48 \%)\end{array}$ & 4,535 & 73 & 1037.24 & $\begin{array}{l}1.61 \% \\
(1.26 \% \\
2.02 \%\end{array}$ \\
\hline \multicolumn{13}{|l|}{ Gender } \\
\hline Male & 20,404 & 64 & 256.25 & $\begin{array}{l}0.31 \% \\
(0.24 \% \\
0.40 \%)\end{array}$ & 20,405 & 96 & 1680.10 & $\begin{array}{l}0.47 \% \\
(0.38 \% \\
0.57 \%)\end{array}$ & 20,405 & 129 & 4681.68 & $\begin{array}{l}0.63 \% \\
(0.53 \% \\
0.75 \%\end{array}$ \\
\hline Female & 22,325 & 62 & 293.61 & $\begin{array}{l}0.28 \% \\
(0.21 \% \\
0.36 \%)\end{array}$ & 22,326 & 90 & 1842.21 & $\begin{array}{l}0.40 \% \\
(0.32 \% \\
0.50 \%)\end{array}$ & 22,327 & 123 & 5148.73 & $\begin{array}{l}0.55 \% \\
(0.46 \% \\
0.66 \%\end{array}$ \\
\hline Unknown & 13 & 0 & 0.17 & $\begin{array}{l}0.00 \% \\
(0.00 \% \\
0.00 \%)\end{array}$ & 13 & 0 & 1.10 & $\begin{array}{l}0.00 \% \\
(0.00 \% \\
0.00 \%)\end{array}$ & 13 & 0 & 2.91 & $\begin{array}{l}0.00 \% \\
(0.00 \% \\
0.00 \%\end{array}$ \\
\hline \multicolumn{13}{|l|}{ Race } \\
\hline White & 38,246 & 113 & 487.13 & $\begin{array}{l}0.30 \% \\
(0.24 \% \\
0.36 \%)\end{array}$ & 38,248 & 160 & 3154.63 & $\begin{array}{l}0.42 \% \\
(0.36 \% \\
0.49 \%)\end{array}$ & 38,249 & 218 & 8814.21 & $\begin{array}{l}0.57 \% \\
(0.50 \% \\
0.65 \%\end{array}$ \\
\hline $\begin{array}{l}\text { Black or } \\
\text { African } \\
\text { American }\end{array}$ & 2,545 & 9 & 36.89 & $\begin{array}{l}0.35 \% \\
(0.16 \%, \\
0.67 \%)\end{array}$ & 2,545 & 17 & 210.81 & $\begin{array}{l}0.67 \% \\
(0.39 \%, \\
1.07 \%)\end{array}$ & 2,545 & 23 & 585.70 & $\begin{array}{l}0.90 \% \\
(0.57 \% \\
1.35 \%\end{array}$ \\
\hline Asian & 212 & 0 & 3.33 & $\begin{array}{l}0.00 \% \\
(0.00 \% \\
0.00 \%)\end{array}$ & 212 & 0 & 17.43 & $\begin{array}{l}0.00 \% \\
(0.00 \% \\
0.00 \%)\end{array}$ & 212 & 1 & 49.07 & $\begin{array}{l}0.47 \% \\
(0.01 \% \\
2.60 \%\end{array}$ \\
\hline Other/Unknown & 1,739 & 4 & 22.68 & $\begin{array}{l}0.23 \% \\
(0.06 \% \\
0.59 \%)\end{array}$ & 1,739 & 9 & 140.54 & $\begin{array}{l}0.52 \% \\
(0.24 \% \\
0.98 \%)\end{array}$ & 1,739 & 10 & 384.34 & $\begin{array}{l}0.58 \% \\
(0.28 \% \\
1.06 \%\end{array}$ \\
\hline \multicolumn{13}{|l|}{$\begin{array}{l}\text { Type } 1 \\
\text { Diabetes during } \\
\text { baseline }\end{array}$} \\
\hline Yes & 270 & 1 & 3.85 & $\begin{array}{l}0.37 \% \\
(0.01 \% \\
2.05 \%)\end{array}$ & 270 & 2 & 22.56 & $\begin{array}{l}0.74 \% \\
(0.09 \% \\
2.65 \%)\end{array}$ & 271 & 3 & 64.50 & $\begin{array}{l}1.11 \% \\
(0.23 \% \\
3.20 \%\end{array}$ \\
\hline No & 42,472 & 125 & 546.17 & $\begin{array}{l}0.29 \% \\
(0.25 \% \\
0.35 \%)\end{array}$ & 42,474 & 184 & 3500.85 & $\begin{array}{l}0.43 \% \\
(0.37 \% \\
0.50 \%)\end{array}$ & 42,474 & 249 & 9768.82 & $\begin{array}{l}0.59 \% \\
(0.52 \% \\
0.66 \%\end{array}$ \\
\hline
\end{tabular}

Type 2

Diabetes during

baseline 


\begin{tabular}{|c|c|c|c|c|c|c|c|c|c|c|c|c|}
\hline \multirow{2}{*}{$\begin{array}{l}\text { Stroke } \\
\text { incidence type }\end{array}$} & \multicolumn{4}{|c|}{ During index hospitalization } & \multicolumn{4}{|c|}{ Up to 30 days post-operation } & \multicolumn{4}{|c|}{ Up to 90 days post-operation } \\
\hline & $\begin{array}{l}\text { Number } \\
\text { of } \\
\text { patients } \\
\text { at risk }\end{array}$ & $\begin{array}{l}\text { Number } \\
\text { of } \\
\text { cases }\end{array}$ & $\begin{array}{l}\text { Years } \\
\text { at risk }\end{array}$ & $\begin{array}{l}\text { Incidence } \\
\text { proportion } \\
\text { (95\% } \\
\text { confidence } \\
\text { interval) }\end{array}$ & $\begin{array}{l}\text { Number } \\
\text { of } \\
\text { patients } \\
\text { at risk }\end{array}$ & $\begin{array}{l}\text { Number } \\
\text { of } \\
\text { cases }\end{array}$ & $\begin{array}{l}\text { Years at } \\
\text { risk }\end{array}$ & $\begin{array}{l}\text { Incidence } \\
\text { proportion } \\
\text { (95\% } \\
\text { confidence } \\
\text { interval) }\end{array}$ & $\begin{array}{l}\text { Number } \\
\text { of } \\
\text { patients } \\
\text { at risk }\end{array}$ & $\begin{array}{l}\text { Number } \\
\text { of } \\
\text { cases }\end{array}$ & $\begin{array}{l}\text { Years at } \\
\text { risk }\end{array}$ & $\begin{array}{l}\text { Incide } \\
\text { propol } \\
\text { (95\% } \\
\text { confid } \\
\text { interv: }\end{array}$ \\
\hline Yes & 5,923 & 23 & 82.24 & $\begin{array}{l}0.39 \% \\
(0.25 \% \\
0.58 \%)\end{array}$ & 5,923 & 42 & 492.66 & $\begin{array}{l}0.71 \% \\
(0.51 \% \\
0.96 \%)\end{array}$ & 5,924 & 58 & 1394.58 & $\begin{array}{l}0.98 \% \\
(0.74 \% \\
1.26 \%\end{array}$ \\
\hline No & 36,819 & 103 & 467.78 & $\begin{array}{l}0.28 \% \\
(0.23 \%, \\
0.34 \%)\end{array}$ & 36,821 & 144 & 3030.75 & $\begin{array}{l}0.39 \% \\
(0.33 \% \\
0.46 \%)\end{array}$ & 36,821 & 194 & 8438.74 & $\begin{array}{l}0.53 \% \\
(0.46 \% \\
0.61 \%\end{array}$ \\
\hline \multicolumn{13}{|l|}{$\begin{array}{l}\text { Existing } \\
\text { permanently } \\
\text { implanted } \\
\text { device or } \\
\text { prosthesis at } \\
\text { baseline }\end{array}$} \\
\hline Yes & 1,242 & 0 & 17.80 & $\begin{array}{l}0.00 \% \\
(0.00 \%, \\
0.00 \%)\end{array}$ & 1,242 & 1 & 102.71 & $\begin{array}{l}0.08 \% \\
(0.00 \% \\
0.45 \%)\end{array}$ & 1,242 & 1 & 287.33 & $\begin{array}{l}0.08 \% \\
(0.00 \% \\
0.45 \%\end{array}$ \\
\hline No & 41,500 & 126 & 532.23 & $\begin{array}{l}0.30 \% \\
(0.25 \% \\
0.36 \%)\end{array}$ & 41,502 & 185 & 3420.70 & $\begin{array}{l}0.45 \% \\
(0.38 \% \\
0.51 \%)\end{array}$ & 41,503 & 251 & 9545.98 & $\begin{array}{l}0.60 \% \\
(0.53 \% \\
0.68 \%\end{array}$ \\
\hline \multicolumn{13}{|l|}{$\begin{array}{l}\text { Total length of } \\
\text { hospital stay }\end{array}$} \\
\hline $1-5$ days & 37,077 & 60 & 393.57 & $\begin{array}{l}0.16 \% \\
(0.12 \% \\
0.21 \%)\end{array}$ & 37,079 & 104 & 3053.66 & $\begin{array}{l}0.28 \% \\
(0.23 \% \\
0.34 \%)\end{array}$ & 37,080 & 148 & 8514.74 & $\begin{array}{l}0.40 \% \\
(0.34 \% \\
0.47 \%\end{array}$ \\
\hline $6-10$ days & 4,530 & 43 & 98.86 & $\begin{array}{l}0.95 \% \\
(0.69 \% \\
1.28 \%)\end{array}$ & 4,530 & 57 & 375.63 & $\begin{array}{l}1.26 \% \\
(0.95 \%, \\
1.63 \%)\end{array}$ & 4,530 & 70 & 1054.52 & $\begin{array}{l}1.55 \% \\
(1.21 \% \\
1.95 \%\end{array}$ \\
\hline$>10$ days & 1,135 & 23 & 57.60 & $\begin{array}{l}2.03 \% \\
(1.29 \%, \\
3.03 \%)\end{array}$ & 1,135 & 25 & 94.11 & $\begin{array}{l}2.20 \% \\
(1.43 \% \\
3.23 \%)\end{array}$ & 1,135 & 34 & 264.06 & $\begin{array}{l}3.00 \% \\
(2.08 \% \\
4.16 \%\end{array}$ \\
\hline \multicolumn{13}{|l|}{$\begin{array}{l}\text { Use of } \\
\text { implanted } \\
\text { material during } \\
\text { surgery on the } \\
\text { same day as } \\
\text { index surgery }\end{array}$} \\
\hline Yes & 3,095 & 11 & 44.45 & $\begin{array}{l}0.36 \% \\
(0.18 \% \\
0.64 \%)\end{array}$ & 3,095 & 16 & 255.45 & $\begin{array}{l}0.52 \% \\
(0.30 \% \\
0.84 \%)\end{array}$ & 3,095 & 19 & 714.26 & $\begin{array}{l}0.61 \% \\
(0.37 \% \\
0.96 \%\end{array}$ \\
\hline No & 39,647 & 115 & 505.58 & $\begin{array}{l}0.29 \% \\
(0.24 \% \\
0.35 \%)\end{array}$ & 39,649 & 170 & 3267.96 & $\begin{array}{l}0.43 \% \\
(0.37 \% \\
0.50 \%)\end{array}$ & 39,650 & 233 & 9119.06 & $\begin{array}{l}0.59 \% \\
(0.51 \% \\
0.67 \%\end{array}$ \\
\hline \multicolumn{13}{|l|}{$\begin{array}{l}\text { Medical history } \\
\text { of stroke }\end{array}$} \\
\hline Yes & 291 & 39 & 4.59 & $\begin{array}{l}13.40 \% \\
(9.71 \%, \\
17.86 \%)\end{array}$ & 292 & 58 & 20.47 & $\begin{array}{l}19.86 \% \\
(15.44 \% \\
24.91 \%)\end{array}$ & 292 & 77 & 54.44 & $\begin{array}{l}26.37 \\
(21.41 \\
31.82\end{array}$ \\
\hline No & 42,451 & 87 & 545.43 & $\begin{array}{l}0.20 \% \\
(0.16 \% \\
0.25 \%)\end{array}$ & 42,452 & 128 & 3502.94 & $\begin{array}{l}0.30 \% \\
(0.25 \% \\
0.36 \%)\end{array}$ & 42,453 & 175 & 9778.87 & $\begin{array}{l}0.41 \% \\
(0.35 \% \\
0.48 \%\end{array}$ \\
\hline
\end{tabular}


Table 3

Incidence rate of stroke in patients undergoing elective posterior lumbar fusion

\begin{tabular}{|c|c|c|c|c|c|c|c|c|c|c|c|c|}
\hline \multirow{2}{*}{$\begin{array}{l}\text { Stroke } \\
\text { incidence type }\end{array}$} & \multicolumn{4}{|c|}{ During index hospitalization } & \multicolumn{4}{|c|}{ Up to 30 days post-operation } & \multicolumn{4}{|c|}{ Up to 90 days post-operation } \\
\hline & $\begin{array}{l}\text { Number } \\
\text { of } \\
\text { patients } \\
\text { at risk }\end{array}$ & $\begin{array}{l}\text { Number } \\
\text { of } \\
\text { cases }\end{array}$ & $\begin{array}{l}\text { Years } \\
\text { at risk }\end{array}$ & $\begin{array}{l}\text { Incidence } \\
\text { rate per } \\
1000 \\
\text { years } \\
\text { (95\% } \\
\text { confidence } \\
\text { interval) }\end{array}$ & $\begin{array}{l}\text { Number } \\
\text { of } \\
\text { patients } \\
\text { at risk }\end{array}$ & $\begin{array}{l}\text { Number } \\
\text { of } \\
\text { cases }\end{array}$ & $\begin{array}{l}\text { Years at } \\
\text { risk }\end{array}$ & $\begin{array}{l}\text { Incidence } \\
\text { rate per } \\
1000 \\
\text { years } \\
\text { (95\% } \\
\text { confidence } \\
\text { interval) }\end{array}$ & $\begin{array}{l}\text { Number } \\
\text { of } \\
\text { patients } \\
\text { at risk }\end{array}$ & $\begin{array}{l}\text { Number } \\
\text { of } \\
\text { cases }\end{array}$ & $\begin{array}{l}\text { Years at } \\
\text { risk }\end{array}$ & $\begin{array}{l}\text { Incide } \\
\text { rate pi } \\
1000 \\
\text { years } \\
(95 \% \\
\text { confid } \\
\text { intervi }\end{array}$ \\
\hline Crude & 42,742 & 126 & 550.03 & $\begin{array}{l}229.08 \\
(192.38 \\
272.78)\end{array}$ & 42,744 & 186 & 3523.41 & $\begin{array}{l}52.79 \\
(45.72 \\
60.95)\end{array}$ & 42,745 & 252 & 9833.32 & $\begin{array}{l}25.63 \\
(22.65 \\
28.99)\end{array}$ \\
\hline
\end{tabular}

Strafications

by:

Age

$\begin{array}{llll}>=18-55 & 15,181 & 17 & 179.62\end{array}$

94.64

$(58.83$

$15,181 \quad 25$

$1251.95 \quad 19.97$

(13.49,

$15,181 \quad 36$

$3498.67 \quad 10.29$

152.24)

29.55)

$14.26)$

$56-65$

$\begin{array}{llll}11,588 & 22 & 146.04 & 150.64\end{array}$

$(99.19$

$\begin{array}{llll}11,590 & 39 & 956.04 & 40.79\end{array}$

(29.80,

228.78)

$55.83)$

$\begin{array}{llll}11,591 & 53 & 2662.86 & 19.90\end{array}$

19.90
$(15.21$

$\begin{array}{ll}157.62 \quad 323.56 \\ & (245.90,\end{array}$

$11,438 \quad 71$

$942.84 \quad 75.30$

26.05)

$66-75$

$\begin{array}{llll}11,438 & 51 & 157.62 & 323.56 \\ & & & (245.90, \\ & & 425.74)\end{array}$

$76-<86$

$4,535 \quad 36$

$66.74 \quad 539.42$

539.42
747.82)

$4,535 \quad 51$

(59.68,

95.02)

$11,438 \quad 90$

$2634.55 \quad 34.16$

34.16
$(27.79$

42.00)

$372.57 \quad 136.89$

$(104.03$,
$180.12)$

$4,535 \quad 73$

$1037.24 \quad 70.38$

(55.95

Gender

Male

$20,404 \quad 64$

256.25

$249.76 \quad 20,405 \quad 96$

(195.49,

319.10)

Female

$22,325 \quad 62$

$293.61 \quad 211.16$

211.16
$(164.63$

270.84)

1680.10

57.14

20,405

$\begin{array}{lllll}57.14 & 20,405 & 129 & 4681.68 & 27.55\end{array}$

(46.78,

69.79)

32.74)

$\begin{array}{llll}22,326 & 90 & 1842.21 & 48.85\end{array}$

48.85
$(39.74$
$60.07)$

22,327

$5148.73 \quad 23.89$

60.07)

28.51)

Unknown

130

0.17

$0.00(0.00, \quad 13$
$0.00)$

1.10

$0.00(0.00$

2.91

$0.00(1$

Race

White

38,246

487.13

38

(1)

\begin{tabular}{|c|c|c|c|c|c|c|c|c|c|c|c|c|}
\hline $\begin{array}{l}\text { Black or } \\
\text { African } \\
\text { American }\end{array}$ & 2,545 & 9 & 36.89 & $\begin{array}{l}243.99 \\
(126.95 \\
468.92)\end{array}$ & 2,545 & 17 & 210.81 & $\begin{array}{l}80.64 \\
(50.13 \\
129.72)\end{array}$ & 2,545 & 23 & 585.70 & $\begin{array}{l}39.27 \\
(26.10 \\
59.09)\end{array}$ \\
\hline Asian & 212 & 0 & 3.33 & $\begin{array}{l}0.00(0.00, \\
0.00)\end{array}$ & 212 & 0 & 17.43 & $\begin{array}{l}0.00(0.00, \\
0.00)\end{array}$ & 212 & 1 & 49.07 & $\begin{array}{l}20.38 \\
(2.87 \\
144.6 !\end{array}$ \\
\hline Other/Unknown & 1,739 & 4 & 22.68 & $\begin{array}{l}176.39 \\
(66.20 \\
469.96)\end{array}$ & 1,739 & 9 & 140.54 & $\begin{array}{l}64.04 \\
(33.32 \\
123.08)\end{array}$ & 1,739 & 10 & 384.34 & $\begin{array}{l}26.02 \\
(14.00 \\
48.36)\end{array}$ \\
\hline
\end{tabular}

Type 1

Diabetes during

baseline

\begin{tabular}{|c|c|c|c|c|c|c|c|c|c|c|c|c|}
\hline Yes & 270 & 1 & 3.85 & $\begin{array}{l}259.59 \\
(36.57 \\
1,842.88)\end{array}$ & 270 & 2 & 22.56 & $\begin{array}{l}88.64 \\
(22.17 \\
354.43)\end{array}$ & 271 & 3 & 64.50 & $\begin{array}{l}46.51 \\
(15.00 \\
144.2:\end{array}$ \\
\hline No & 42,472 & 125 & 546.17 & $\begin{array}{l}228.86 \\
(192.06 \\
272.72)\end{array}$ & 42,474 & 184 & 3500.85 & $\begin{array}{l}52.56 \\
(45.49 \\
60.73)\end{array}$ & 42,474 & 249 & 9768.82 & $\begin{array}{l}25.49 \\
(22.51 \\
28.86)\end{array}$ \\
\hline
\end{tabular}

Type 2

Diabetes during

baseline 


\begin{tabular}{|c|c|c|c|c|c|c|c|c|c|c|c|c|}
\hline \multirow{2}{*}{$\begin{array}{l}\text { Stroke } \\
\text { incidence type }\end{array}$} & \multicolumn{4}{|c|}{ During index hospitalization } & \multicolumn{4}{|c|}{ Up to 30 days post-operation } & \multicolumn{4}{|c|}{ Up to 90 days post-operation } \\
\hline & $\begin{array}{l}\text { Number } \\
\text { of } \\
\text { patients } \\
\text { at risk }\end{array}$ & $\begin{array}{l}\text { Number } \\
\text { of } \\
\text { cases }\end{array}$ & $\begin{array}{l}\text { Years } \\
\text { at risk }\end{array}$ & $\begin{array}{l}\text { Incidence } \\
\text { rate per } \\
1000 \\
\text { years } \\
(95 \% \\
\text { confidence } \\
\text { interval) }\end{array}$ & $\begin{array}{l}\text { Number } \\
\text { of } \\
\text { patients } \\
\text { at risk }\end{array}$ & $\begin{array}{l}\text { Number } \\
\text { of } \\
\text { cases }\end{array}$ & $\begin{array}{l}\text { Years at } \\
\text { risk }\end{array}$ & $\begin{array}{l}\text { Incidence } \\
\text { rate per } \\
1000 \\
\text { years } \\
\text { (95\% } \\
\text { confidence } \\
\text { interval) }\end{array}$ & $\begin{array}{l}\text { Number } \\
\text { of } \\
\text { patients } \\
\text { at risk }\end{array}$ & $\begin{array}{l}\text { Number } \\
\text { of } \\
\text { cases }\end{array}$ & $\begin{array}{l}\text { Years at } \\
\text { risk }\end{array}$ & $\begin{array}{l}\text { Incide } \\
\text { rate pi } \\
1000 \\
\text { years } \\
(95 \% \\
\text { confid } \\
\text { interv: }\end{array}$ \\
\hline Yes & 5,923 & 23 & 82.24 & $\begin{array}{l}279.66 \\
(185.84 \\
420.84)\end{array}$ & 5,923 & 42 & 492.66 & $\begin{array}{l}85.25 \\
(63.00 \\
115.36)\end{array}$ & 5,924 & 58 & 1394.58 & $\begin{array}{l}41.59 \\
(32.15 \\
53.80)\end{array}$ \\
\hline No & 36,819 & 103 & 467.78 & $\begin{array}{l}220.19 \\
(181.52 \\
267.09)\end{array}$ & 36,821 & 144 & 3030.75 & $\begin{array}{l}47.51 \\
(40.35 \\
55.94)\end{array}$ & 36,821 & 194 & 8438.74 & $\begin{array}{l}22.99 \\
(19.97 \\
26.46)\end{array}$ \\
\hline \multicolumn{13}{|l|}{$\begin{array}{l}\text { Existing } \\
\text { permanently } \\
\text { implanted } \\
\text { device or } \\
\text { prosthesis } \\
\text { during baseline }\end{array}$} \\
\hline Yes & 1,242 & 0 & 17.80 & $\begin{array}{l}0.00(0.00, \\
0.00)\end{array}$ & 1,242 & 1 & 102.71 & $\begin{array}{l}9.74(1.37 \\
69.12)\end{array}$ & 1,242 & 1 & 287.33 & $\begin{array}{l}3.48(( \\
24.71)\end{array}$ \\
\hline No & 41,500 & 126 & 532.23 & $\begin{array}{l}236.74 \\
(198.81 \\
281.91)\end{array}$ & 41,502 & 185 & 3420.70 & $\begin{array}{l}54.08 \\
(46.82 \\
62.47)\end{array}$ & 41,503 & 251 & 9545.98 & $\begin{array}{l}26.29 \\
(23.23 \\
29.76)\end{array}$ \\
\hline \multicolumn{13}{|l|}{$\begin{array}{l}\text { Total length of } \\
\text { hospital stay }\end{array}$} \\
\hline $1-5$ days & 37,077 & 60 & 393.57 & $\begin{array}{l}152.46 \\
(118.38, \\
196.36)\end{array}$ & 37,079 & 104 & 3053.66 & $\begin{array}{l}34.07 \\
(28.11 \\
41.28)\end{array}$ & 37,080 & 148 & 8514.74 & $\begin{array}{l}17.39 \\
(14.80 \\
20.42)\end{array}$ \\
\hline $6-10$ days & 4,530 & 43 & 98.86 & $\begin{array}{l}434.95 \\
(322.58 \\
586.48)\end{array}$ & 4,530 & 57 & 375.63 & $\begin{array}{l}151.74 \\
(117.05 \\
196.72)\end{array}$ & 4,530 & 70 & 1054.52 & $\begin{array}{l}66.38 \\
(52.52 \\
83.90)\end{array}$ \\
\hline$>10$ days & 1,135 & 23 & 57.60 & $\begin{array}{l}399.33 \\
(265.37 \\
600.93)\end{array}$ & 1,135 & 25 & 94.11 & $\begin{array}{l}265.64 \\
(179.49 \\
393.12)\end{array}$ & 1,135 & 34 & 264.06 & $\begin{array}{l}128.71 \\
(92.00 \\
180.21\end{array}$ \\
\hline \multicolumn{13}{|l|}{$\begin{array}{l}\text { Use of } \\
\text { implanted } \\
\text { material during } \\
\text { surgery on the } \\
\text { same day as } \\
\text { index surgery }\end{array}$} \\
\hline Yes & 3,095 & 11 & 44.45 & $\begin{array}{l}247.47 \\
(137.05 \\
446.87)\end{array}$ & 3,095 & 16 & 255.45 & $\begin{array}{l}62.63 \\
(38.37 \\
102.24)\end{array}$ & 3,095 & 19 & 714.26 & $\begin{array}{l}26.60 \\
(16.97 \\
41.70)\end{array}$ \\
\hline No & 39,647 & 115 & 505.58 & $\begin{array}{l}227.46 \\
(189.47 \\
273.08)\end{array}$ & 39,649 & 170 & 3267.96 & $\begin{array}{l}52.02 \\
(44.76 \\
60.46)\end{array}$ & 39,650 & 233 & 9119.06 & $\begin{array}{l}25.55 \\
(22.47 \\
29.05)\end{array}$ \\
\hline \multicolumn{13}{|l|}{$\begin{array}{l}\text { Medical history } \\
\text { of stroke }\end{array}$} \\
\hline Yes & 291 & 39 & 4.59 & $\begin{array}{l}8,494.19 \\
(6,206.12 \\
11,625.81)\end{array}$ & 292 & 58 & 20.47 & $\begin{array}{l}2,833.29 \\
(2,190.40 \\
3,664.87)\end{array}$ & 292 & 77 & 54.44 & $\begin{array}{l}1,414 . \\
(1,131 \\
1,786 .\end{array}$ \\
\hline No & 42,451 & 87 & 545.43 & $\begin{array}{l}159.51 \\
(129.28 \\
196.80)\end{array}$ & 42,452 & 128 & 3502.94 & $\begin{array}{l}36.54 \\
(30.73 \\
43.45)\end{array}$ & 42,453 & 175 & 9778.87 & $\begin{array}{l}17.90 \\
(15.43 \\
20.75)\end{array}$ \\
\hline
\end{tabular}

[Insert Table 2 here]

Stratified analyses revealed that older patients consistently had higher incidences of post-operative stroke during all surgical risk windows. For example, the IR of post-operative stroke per $1000 \mathrm{PYs} \leq 365$ days post-operation was 4.87 ( $95 \% \mathrm{Cl}: 3.79,6.25)$ among those aged $\geq 18$ to 55 years, 11.12 (95\% Cl: $9.18,13.46$ ) among those aged 56 to 65 years, 20.25 (95\% Cl: 17.56, 23.34) among those aged 66 to 75 years, and 33.41 (95\% Cl: 28.02, 39.84) among those aged 76 to < 86 years. Moreover, men had higher incidences of stroke than women; for instance, the IR of post-operative stroke per 1000 PYs during index hospitalization was 249.76 (95\% Cl: 195.49, 319.10) for men and 211.16 (95\% Cl: $164.63,270.84)$ for women. Additionally, black patients had higher stroke incidences than 
white, Asian, or other patients: the IR of post-operative stroke per 1000 PYs $\leq 365$ days post-operation was 13.29 (95\% Cl: 12.08, 14.63) among white adults, 20.36 (95\% Cl: $15.05,27.55)$ among black adults, 17.43 (95\% Cl: 5.62, 54.04) among Asian adults, and 12.11 (95\% Cl: 7.42, 19.76) among other adults.

[Insert Table 3 here]

The incidence of post-operative stroke was higher among patients with a history of type 2 diabetes than among patients without such history; for instance, the IR per 1000 PYs of stroke was 24.47 (95\% Cl: $20.51,29.20)$ for those with a history of type 2 diabetes and 11.88 (95\% Cl: $10.71,13.18)$ for those without such history $\leq 365$ days post-operation. The incidence of post-operative stroke was much higher among patients with a history of stroke than among patients without a history of stroke during all risk windows. For example, the IR of post-operative stroke per 1000 PYs during index hospitalization was 8,494.19 (95\% Cl: 6,206.12, 11,625.81) among those with a history of stroke and 159.51 (95\% Cl: 129.28, 196.80) among those without such history. Lastly, patients with longer hospital stays had a higher incidence of stroke. However, it should be emphasized that these longer hospital stays could be a result of stroke instead of a reverse relationship (i.e., where stroke is the consequence of a longer hospital stay).

\section{Discussion}

\section{Stroke incidence}

Minhas et al. examined the incidence of peri-operative cerebrovascular accidents (CVAs) among patients undergoing elective orthopedic procedures from 2006 to 2012 within the National Surgical Quality Improvement Program (NSQIP) database and reported that the 30-day IP of CVA was 0.35\% for singlelevel/multilevel PLF $(n=2,895)$ [9]. In our study, a 30 -day IP for post-operative stroke of $0.44 \%(95 \% \mathrm{Cl}: 0.38 \%$, $0.50 \%)$ was observed; however, it should be noted the authors defined their procedures using Current Procedure Terminology (CPT) codes and their definition for CVA was based on medical record review [9]. In a 2012 retrospective database study, 2015 PLF patients were identified from a nationwide Taiwanese cohort from 2000 to 2005 using The National Health Insurance Research Database and followed up for three years. The IR of stroke per 1000 PYs was 10.22 (95\% Cl: 7.94, 13.17). In their study, Wu et al. used ICD-9-CM codes 430-435 for stroke and ICD-9-PCS codes 81.0 and 81.38 to identify PLF [10]. The IR of stroke per 1000 PYs in our study was 13.68 (95\% Cl: $12.51,14.96) \leq 365$ days post-operation, which was in line with their finding.

A 2014 retrospective cohort study performed by Marquez-Lara et al. used the Nationwide Inpatient Sample (NIS) database from 2002 to 2011 to identify patients undergoing elective lumbar fusion procedures and found a post-operative CVA incidence of $0.15 \%$ among the PLF patients ( $\mathrm{n}=214,837$ ) [11]. In our study, the IP of stroke following elective PLF ranged from $0.29 \%$ ( $95 \% \mathrm{Cl}: 0.25 \%, 0.35 \%)$ to $1.12 \%(95 \% \mathrm{Cl}: 1.03 \%$, $1.23 \%)$. It should be noted that only patients with ICD-9 code 81.08 were included and that CVA was defined with only one ICD-9 code (997.02) in their study. However, the researchers also found that increased length of hospital stay was associated with post-operative CVA [11]. Furthermore, a variety of studies examined 30-day outcomes following lumbar spinal fusion within both the NIS and NSQIP databases and generally found that the 30-day overall incidence of post-operative stroke was about $0.20 \%$ [5-8, 12].

Our finding that the risk of stroke increased with age was consistent with the literature within the general population [13]. Furthermore, studies evaluating the risk of stroke among adults undergoing lumbar spinal fusion also found an association between increased age and the risk of stroke [9, 11, 14]. With regards to sex, Minhas et al. and Marquez-Lara et al. found no significant difference in the sex-specific incidence of stroke [9, 11], which is in line with our findings. Stratified analyses revealed that black patients had a higher stroke incidence compared to other ethnic groups. This finding is consistent with the literature as numerous studies have illustrated that African-Americans have significantly higher risk of stroke in the general population [15-17]. Among populations undergoing elective PLF though, there was no published data on the incidence of post-operative stroke by race.

With regards to the other subgroups, limited information among PLF patients is available in the literature. However, Minhas et al. reported that patients with insulin-dependent diabetes mellitus (IDDM) had 3.08 times the odds of a post-operative CVA compared to those without IDDM [9]. Furthermore, recent reviews have demonstrated that diabetes mellitus is an established risk factor for stroke in the general population [18-19]. Thus, our finding that post-operative stroke incidence was higher among patients with a history of type 2 diabetes is consistent with the literature. Lastly, it is known that the incidence of stroke among those with a medical history of stroke is increased (i.e., there is a high risk of recurrent stroke) [18-19]; our results are thus in line with the standard medical knowledge regarding recurrent stroke.

\section{Variable and database considerations}

A 2012 review by Andrade et al. examined the validity of algorithms for identifying CVAs using administrative/claims data among 35 identified studies and ultimately concluded that the algorithms and definitions used to identify CVAs using administrative/claims data differ greatly in the published literature. However, the authors determined that studies reported the highest positive predictive values for inpatient ICD-9 codes $430 . x, 431 . x, 434 . x$, and $436 . x$ for acute stroke while algorithms that included ICD-9 codes 433.x1, 434 (excluding 434.x0), and 436 performed well (85\% or higher) for transient ischemic stroke [20].

Lastly, it is noteworthy that our study used Optum EHR while most studies in the literature used NSQIP or NIS. Because of differences in these data sources, it may not be surprising that our results would not exactly align with the incidence information found in the literature. For instance, a 2016 study evaluated the variability in standard outcomes of PLF between the University HealthSystem Consortium (UHC) and the NIS and found that the databases had similar patient populations undergoing PLF, but that the UHC database reported significantly higher complication rates and longer lengths of hospital [21]. Additionally, recent studies have also shown that certain variables have changed over time within both NIS and NSQIP [8, 14]; thus, even comparisons within the same database can be fraught.

\section{Strengths and limitations}


The Optum EHR database has both inpatient and outpatient data as well as a large sample size that enabled us to generate real-world incidence estimates that are generalizable to a segment of the commercially insured US population (i.e., those in the Optum network). Like other studies utilizing secondary data sources without validation (e.g., medical chart review), exposure and outcome misclassification are possible; diagnosis codes may have been incorrect or included as part of the diagnostic rule-out process rather than an indication of disease or surgery itself. Furthermore, patients may have sought healthcare outside Optum EHR prior to the index surgery, so it is possible that a patient developed a stroke prior to the index surgery; similarly, some incident events may have been missed if a patient sought care outside the system after surgery. Additionally, as we used a broad set of ICD codes to identify stroke events, we may have overestimated its incidence.

\section{Conclusion}

This study estimated the incidence of stroke using an EHR database among adults undergoing elective PLF during various post-operative risk windows and among different subgroups. This incidence is slightly higher than that reported in the literature; however, the discrepancy is due to differences in the variable definitions, study populations, follow-up periods, and data sources between our study and those in the literature.

\section{List Of Abbreviations}

$\mathrm{Cl}$

CPT

CVA

EHR

ICD

ICD-9-PCS

ICD-9-CM

ICD-10

IDDM

IP

IR

NIS

NSQIP

PLF

PY

$\mathrm{UHC}$

US
Confidence interval

Current Procedure Terminology

Cerebrovascular accident

Electronic healthcare record

International Classification of Diseases

International Classification of Diseases, Ninth Revision, Procedure Classification System

International Classification of Diseases, Ninth Revision, Clinical Modification

International Classification of Diseases, Tenth Revision

Insulin-dependent diabetes mellitus

Incidence proportion

Incidence rate

Nationwide Inpatient Sample

National Surgical Quality Improvement Program

Posterior lumbar fusion

Person-year

University HealthSystem Consortium

United States

\section{Declarations}

Ethics approval and consent to participate

As this study involved anonymized structured data, which according to applicable legal requirements do not contain data subject to privacy laws, obtaining informed consent from patients was not required.

Consent for publication

Not applicable. 
The data that support the findings of this study are available from Optum but restrictions apply to the availability of these data, which were used under license for the current study, and so are not publicly available. Data are however available from the authors upon reasonable request and with permission of Optum.

Competing interests

All authors are employees of Pfizer Inc., New York, NY, USA.

Funding

This work was funded by Pfizer Inc.

Authors' contribution

$\mathrm{PA}, \mathrm{JM}, \mathrm{CS}, \mathrm{EB}, \mathrm{AG}$, and $\mathrm{KH}$ contributed to the study design. $\mathrm{PA}, \mathrm{KH}, \mathrm{XZ}, \mathrm{QL}, \mathrm{RS}$, and $\mathrm{CW}$ contributed to data analyses. PA and $\mathrm{KH}$ drafted the manuscript with input from JM, CS, EB, AG, XZ, QL, RS, and CW. All authors read and approved the final manuscript.

Acknowledgements

Not applicable.

\section{References}

[1] GBD 2016 Stroke Collaborators. Global, regional, and national burden of stroke, 1990-2016: a systematic analysis for the Global Burden of Disease Study 2016. Lancet Neurol. 2019;18(5):439-458. https://doi.org/10.1016/S1474-4422(19)30034-1.

[2] Yang F, Zhao J, Xu H. Characteristics of hemorrhagic stroke following spine and joint surgeries. Biomed Res Int. 2017:5390839. https://doi.org/10.1155/2017/5390839.

[3] Martin BI, Mirza SK, Spina N, Spiker WR, Lawrence B, Brodke DS. Trends in lumbar fusion procedures rates and associated hospital costs for degenerative spinal diseases in the United States, 2004 to 2015. Spine (Phila Pa 1976). 2019;44(5):369-376. https://doi.org/10.1097/BRS.0000000000002822.

[4] Etzioni DA, Liu JH, Maggard MA, Ko CY. The aging population and its impact on the surgery workforce. Ann Surg. 2003;238(2):170-7. https://doi.org/10.1097/01.SLA.0000081085.98792.3d.

[5] Bronheim RS, Oermann EK, Cho SK, Caridi JM. Coagulation profile as a risk factor for 30-day morbidity and mortality following posterior lumbar fusion. Spine (Phila Pa 1976). 2017;42(12):950-957. https://doi.org/10.1097/BRS.0000000000001935.

[6] Chung AS, Campbell D, Waldrop R, Crandall D. Metabolic syndrome and 30-day outcomes in elective lumbar spinal fusion. Spine (Phila Pa 1976). 2018;43(9):661-666. https://doi.org/10.1097/BRS.0000000000002397.

Page 12/14 
[7] Basques BA, Diaz-Collado PJ, Geddes BJ, Samuel AM, Lukasiewicz AM, Webb ML et al. Primary and revision posterior lumbar fusion have similar shortterm complication rates. Spine (Phila Pa 1976). 2016;41(2):E101-6. https://doi.org/10.1097/BRS.0000000000001094.

[8] Shultz BN, Bovonratwet P, Ondeck NT, Ottesen TD, McLynn RP, Grauer JN. Evaluating the effect of growing patient numbers and changing data elements in the National Surgical Quality Improvement Program (NSQIP) database over the years: a study of posterior lumbar fusion outcomes. Spine J.

2018;18(11):1982-1988. https://doi.org/10.1016/j.spinee.2018.03.016.

[9] Minhas SV, Goyal P, Patel AA. What are the risk factors for cerebrovascular accidents after elective orthopaedic surgery? Clin Orthop Relat Res. 2016;474(3):611-8. https://doi.org/10.1007/s11999-015-4496-2.

[10] Wu JC, Chen YC, Liu L, Huang WC, Thien PF, Chen TJ et al. Lumbar spine fusion surgery and stroke: a national cohort study. Eur Spine J. 2012;21(12):2680-7. https://doi.org/10.1007/s00586-012-2405-x.

[11] Marquez-Lara A, Nandyala SV, Fineberg SJ, Singh K. Cerebral vascular accidents after lumbar spine fusion. Spine (Phila Pa 1976). 2014;39(8):673-7. https://doi.org/10.1097/BRS.0000000000000197.

[12] Memstoudis SG, Kirksey M, Ma Y, Chiu L, Mazumdar M, Pumberger M et al. Metabolic syndrome and lumbar spine fusion surgery: epidemiology and perioperative outcomes. Spine (Phila Pa 1976). 2012;37(11):989-95. https://doi.org/10.1097/BRS.0b013e31823a3a13.

[13] Kelly-Hayes M. Influence of age and health behaviors on stroke risk: lessons from longitudinal studies. J Am Geriatr Soc. 2010;58 Suppl 2:S325-8. https://doi.org/10.1111/j.1532-5415.2010.02915.x.

[14] Shen Y, Silverstein JC, Roth S. In-hospital complications and mortality after elective spinal fusion surgery in the united states: a study of the nationwide inpatient sample from 2001 to 2005. J Neruosurg Anesthesiol. 2009;21(1):21-30. https://doi.org/10.1097/ANA.0b013e31818b47e9.

[15] Cruz-Flores S, Rabinstein A, Biller J, Elkind MSV, Griffith P, Gorelick PB et al. Racial-ethnic disparities in stroke care: the American experience: a statement for healthcare professionals from the American Heart Association/American Stroke Association. Stroke. 2011;42:2091-2116.

https://doi.org/10.1161/STR.0b013e3182213e24.

[16] Park JH, Ovbiagele B. Association of black race with recurrent stroke risk. J Neurol Sci. 2016;365:203-206. https://doi.org/10.1016/j.jns.2016.04.012.

[17] Mozaffarian D, Benjamin EJ, Go AS, Arnett DK, Blaha MJ, Cushman M et al. Heart disease and stroke statistics-2015 update: a report from the American Heart Association. Circulation. 2015;131:e29-e322. https://doi.org/10.1161/CIR.0000000000000152.

[18] Hankey GJ. Stroke. Lancet. 2017;11;389(10069):641-654. https://doi.org/10.1016/S0140-6736(16)30962-X.

[19] Isabel C, Calvet D, Mas JL. Stroke prevention. Presse Med. 2016;45(12 Pt 2):e457-e471. . https://doi.org/10.1016/j.lpm.2016.10.009.

[20] Andrade SE, Harrold LR, Tjia J, Cutrona SL, Saczynski, Dodd KS et al. A systematic review of validated methods for identifying cerebrovascular accident or transient ischemic attack using administrative data. Pharmacoepidemiol Drug Saf. 2012;21 Suppl 1:100-28. https://doi.org/10.1002/pds.2312. 
[21] Joseph JR, Smith BW, Park P. Variability in standard outcomes of posterior lumbar fusion determined by national databases. World Neurosurg. 2017;97:236-240. https://doi.org/10.1016/j.wneu.2016.09.117. 